\title{
Radical copolymerization in homogenous medium and emulsion system monomers acrylonitrile/styrene
}

\author{
H. Boussehel \\ Laboratoire of applied chemistry LCA, University of Biskra, BP. 145, 0700 Biskra, Algérie
}

\begin{abstract}
This study examines the radical copolymerization in homogeneous and emulsion of the monomer system acrylonitrile/styrene. These copolymers are of great interest to the plastics industry, because they combine the good mechanical properties and implementation provided by the styrene units in the very high solvent resistance and extreme gas impermeability provided by the acrylonitrile units. The properties of a copolymer are directly related to its composition and distribution of monomer units in its macromolecular chains. Based on the reports of the couple reactivity's of monomers (AN/S) found in the literature, the objective of the work is to provide theoretical simulation (by analytical and numerical integration of the equation of copolymerization): The kinetics of the reaction copolymerization of AN/S in a homogeneous medium and emulsion (drift composition, azeotropic) and the microstructure (distribution of monomer sequences) and the glass transition property of the macromolecular chains instant formed throughout the copolymerization reaction.
\end{abstract}

\section{INTRODUCTION}

The two main axes of orientation of research and development of plastic materials with mixed or new properties relate to their development either with an operation of mixture of homopolymers, or by a simultaneous reaction of two or several monomers of different chemical structures. If the first method seems a priori interesting and easy to implement, it is not easily realizable in practice, because the macromolecules of different chemical structures seldom form miscible mixtures $\left(\Delta G_{\text {mélange }}>0\right)$ causing heterogeneous materials with poor properties. The second method causes on the other hand a material copolymer whose macromolecular chains are made of different monomer reasons dependent between them by covalent bonds. The specific properties to this material copolymer are directly related on its chemical composition and the distribution of the monomer reasons in the chains of copolymer. These parameters are controlled primarily by the fundamental sizes of the reaction of copolymerization which are the reports of reactivity of the monomers which constitute the intrinsic sizes of a couple of monomers given. Copolymerization is thus a process privileged for the engineer to find material more adapted for a given application.

\section{RADICAL COPOLYMERIZATION IN HOMOGENOUS MEDIUM}

From the experimental results found in the literature [1-3] and relating to the reports of reactivity of acrylonitrile and styrene in copolymerization in homogeneous medium (Table 1), we then simulated this copolymerization theoretically by using two methods of integration of the equation of copolymerization, one analytical and the other digital one. We used for our calculations the median values of the reports of reactivity $r_{a}=0.13$ et $r_{s}=0.34$.

The first method of simulation is carried out starting from the integral analytical equation suggested by
Table 1. Reports of reactivity of the couple Acrylonitrile/Styrene in radicalizing copolymerization.

\begin{tabular}{|l|c|c|c|c|}
\hline$r_{a}(A N)$ & 0.12 & 0.13 & 0.13 & 0.13 \\
\hline$r_{s}(S)$ & 0.32 & 0.30 & 0.36 & 0.40 \\
\hline Solvent & $\begin{array}{c}\text { Diméthyl- } \\
\text { formamide }\end{array}$ & $\begin{array}{c}\text { Diméthyl- } \\
\text { formamide }\end{array}$ & $\begin{array}{c}\text { Diméthyl- } \\
\text { formamide }\end{array}$ & masse \\
\hline
\end{tabular}

MEYER [4,5] and DIONISIO [6]; this integral equation is commonly used to follow the drift of composition according to conversion [7-10].

$$
x=1-\left[\frac{f_{a}}{f_{a}(0)}\right]^{\alpha_{a}}\left[\frac{f_{s}}{f_{s}(0)}\right]^{\beta_{a}}\left[\frac{f_{a}(0)-\delta_{a}}{f_{a}-\delta_{a}}\right]^{\gamma} .
$$

The second method of simulation is carried out by a digital calculation of the composition of various copolymers formed all the way along the reaction, starting from the equation of composition in its differential form.

$$
n_{A}=\frac{1+r_{a} X_{A}}{1+r_{S} X_{S}} .
$$

\subsection{Results and discussions}

The reports of reactivity being all the two lower than the unit, the curve of incorporation $F_{a}=f\left(f_{a}\right)$, Figure 1 shows on the one hand the existence of a azeotropic composition $f_{a}($ azéo $)=F_{a}($ azéo $)=43.14 \%$ lower than the équimolaire composition $f_{a}=50 \%$ owing to the fact that $r_{a}$ is lower than $r_{s}$ and on the other hand that the behaviour of the reaction of copolymerization is different on both sides of the azeotropic composition.

If $f_{a}<f_{a}$ (azéo), $F_{a}>f_{a}$ the composition of copolymer is richer in monomer acrylonitrile than the initial reactional medium, the acrylonitrile is consumed more quickly than styrene. On the other hand, if $f_{a}>$ $f_{a}(a z e ́ o), F_{a}<f_{a}$, it is the styrene which is consumed most quickly, the macromolecular chains grow rich more and more out of acrylonitrile at the end of the reaction. 


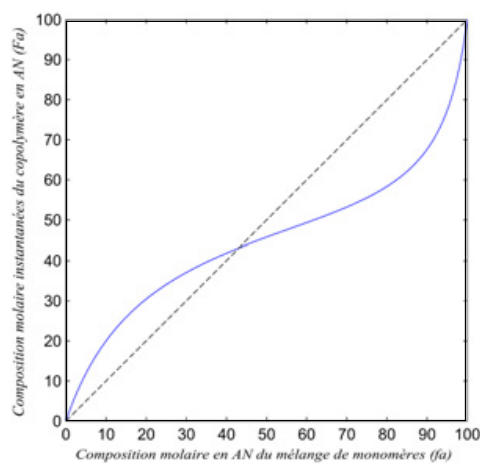

Figure 1. Evolution of $F_{a}$ according to $f_{a}$.

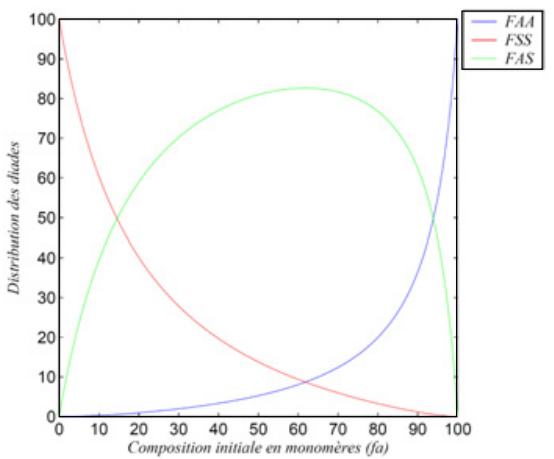

Figure 2. Distribution of the dyads according to $f_{a}$.

The curves of the distribution of the dyads according to the initial composition in monomers according to Figure 2, show that the maximum of the alternate dyads $F_{A S}$ is reached for a composition initially richer in acrylonitrile $f_{a}(0)=62 \%$ what is in agreement with a larger reactivity of styrene $r_{s}=0.34>r_{a}=0.13$; these alternate dyads $F_{A S}$ decrease logically in favour as of dyads of acrylonitrile $F_{A A}$ for the initial compositions higher than $62 \%$ out of acrylonitrile and in favour as of dyads of styrene $F_{S S}$ for the initial compositions lower than this same composition.

\section{RADICAL COPOLYMERIZATION IN EMULSION MEDIUM}

A mathematical treatment by polynomial regression carried out gives us the following equations:

For acrylonitrile:

$$
r_{A}=2 \cdot 5 \cdot 10^{-4}(E / M)^{2}-1 \cdot 3 \cdot 10^{-2}(E / M)+0.13 .
$$

And for styrene:

$$
r_{S}=4 \cdot 3 \cdot 10^{-3}(E / M)^{2}+5 \cdot 3 \cdot 10^{-2}(E / M)+0.34 .
$$

Which analytically makes it possible to have access to the values of $r_{i j}$ for any value of $E / M$ and allow to envisage as many azeotropic compositions in emulsion than the condition of azeotropic is checked $\left(r_{A}\right.$ and $r_{S}$ at the same time lower than the unit), which in the case of our system AN/S corresponds to the field $0 \leq E / M<7,6$ and such as $f_{a}(\text { azéo })_{\text {émulsion }}<f_{a}(\text { azéo })_{\text {masse/solution }}$ (Figure 3).

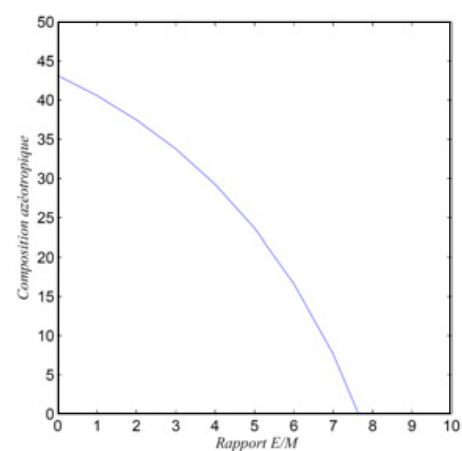

Figure 3. Simulation of copolymerization AN/S. Variation of the azeotropic composition according to the report E/M.

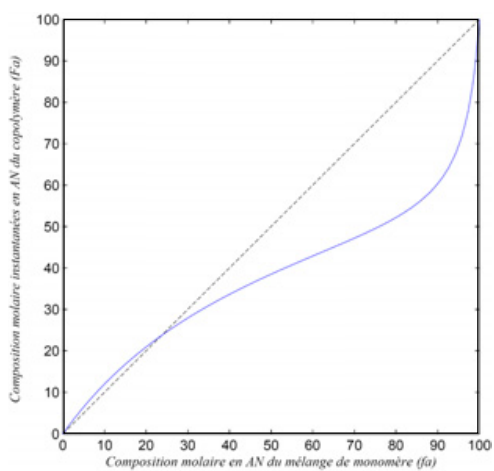

Figure 4. Molar composition of copolymer in $\operatorname{AN}\left(F_{a}\right)$ according to the initial composition in $\mathrm{AN}\left(f_{a}\right)$.

Our theoretical study can be carried out for any report $0<E / M<10$ in emulsion; for the clearness of our work, we chose to present in this memory the study carried out on the emulsions for which $E / M=5$ and $E / M=10$.

- For $E / M=5: r_{a}=0.07, r_{s}=0.71$ Emulsion where there is existence of a azeotropic composition $\left(r_{a}<\right.$ 1 et $\left.r_{s}<1\right)$.

- For $E / M=10: r_{a}=0.025, r_{s}=1.3$ Emulsion where there is no azeotropic composition $\left(r_{a}<1\right.$ et $\left.r_{s}>1\right)$.

\subsection{Emulsion of report $\mathrm{E} / \mathrm{M}=\mathbf{5}$}

The curve of incorporation $F a=f\left(f_{a}\right)$ (Figure 4) a behaviour similar shows us to that of copolymerization in homogeneous medium with the existence of a azeotropic composition, $r_{a}$ and $r_{s}$ being all lower than the unit.

For $f_{a}<f_{a}(a z e ́ o)$ : the copolymer is richer in $\mathrm{AN}$ $\left(F_{a}>f_{a}\right)$.

For $f_{a}>f_{a}(a z e ́ o)$ : it is the styrene which is consumed most quickly $\left(F_{a}<f_{a}\right)$, the copolymer enriched more and more in $\mathrm{AN}$ in the course of reaction. It should be noted however that the value of the azeotropic composition $f_{a}$ (azéo $)_{E / M=5} \approx 23.6 \%$ is weaker than that of copolymerization in homogeneous medium $f_{a}(\text { azéo })_{E / M=0} \approx 43.14 \%$, this is in agreement with a difference in apparent reactivity of the two monomers even more important than in the case of homogeneous copolymerization.

Increase in the reactivity of styrene with the simultaneous reduction in that of acrylonitrile in copolymerization in emulsion for $E / M=5$ compared to their relative 


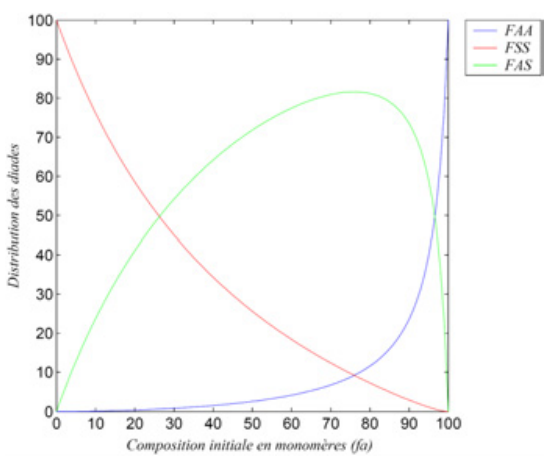

Figure 5. Distribution of the dyads according to the initial composition in monomer.

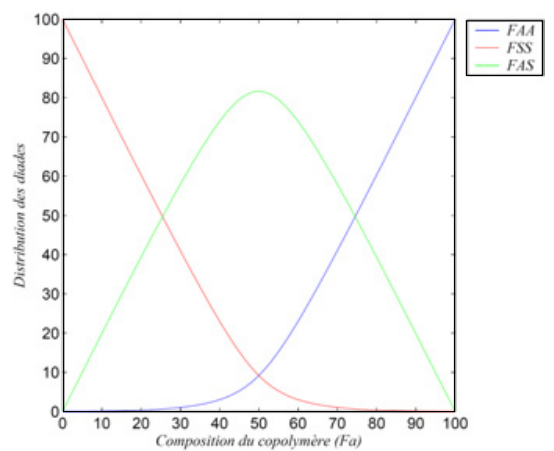

Figure 6. Distribution of the dyads according to the composition of copolymer.

reactivity's in mass/solution $(E / M=0)$ involve obtaining the maximum of alternate dyads $F_{A S}$ for an initial composition of the mixture of the monomers even richer in $\mathrm{AN}\left(f_{a}(0)=75 \%\right)$ (Figure 5).

The distribution of the dyads according to the composition of the copolymer (Figure 6) is very similar to that of copolymerization in homogeneous medium with however a value of the maximum of the alternate dyads always reaches for the copolymer équimolaire, very slightly lower $\left(F_{A S_{(E / M=5)}}=82 \%<F_{A S_{(E / M=0)}}=83 \%\right)$ owing to the fact that the product $r_{a} . r_{s(E / M=5)}=0.050$ that is to say slightly higher than the product $\left.r_{a} \cdot r_{S(E / M=0}\right)=0.044$.

\subsection{Emulsion of report $\mathrm{E} / \mathrm{M}=10$}

As we already noted, the dilution of the emulsion returns the difference in reactivity apparent of the two monomers even more important and in favour of styrene compared to the emulsion more concentrated $(\mathrm{E} / \mathrm{M}=5)$, which had with a larger quantity of AN solubilized in the aqueous phase, which decreases by as much the quantity by $\mathrm{AN}$ in the site real of polymerization (i.e: the organic phase).

Figure 7 , watch that the effect of the dilution of this emulsion is such as styrene is consumed more quickly than acrylonitrile whatever the initial composition of the mixture of the monomers, involving then a composition of copolymer increasingly richer than the initial reactional medium $\left(F_{a}<f_{a} \forall f_{a}(0)\right)$.

It is to be noticed an almost linear evolution of the curve until practically $f_{a}=90 \%$.

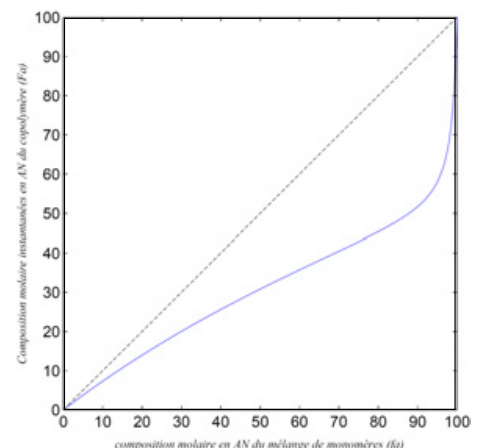

Figure 7. Molar composition instantaneous in AN of copolymer $\left(F_{a}\right)$ according to the molar composition in $\mathrm{AN}$ of the monomer $\left(f_{a}\right)$.

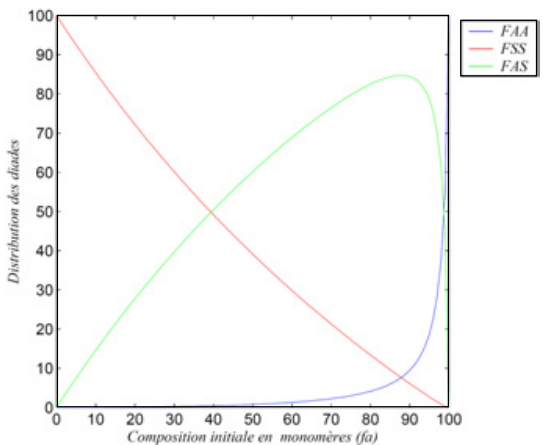

Figure 8. Distribution of the dyads according to the initial composition in monomer.

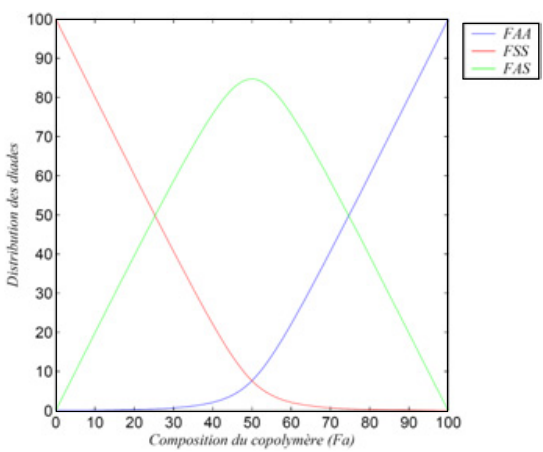

Figure 9. Distribution of the dyads according to the composition of copolymer.

For this emulsion, the low reactivity of the AN comparatively has that obtained for the emulsion with $\mathrm{E} / \mathrm{M}=5$ and with copolymerization in homogeneous medium, involves the existence of very weak fractions of dyads of the type AA (less than $3 \%$ ) to emulsions rich in AN initial $\left(f_{a}(0)=70 \%\right)$ and exceeding the $10 \%$ only starting from the emulsion with more than $90 \%$ of AN; in addition the dyads of the type SS remain in considerable quantity even for initial weak mixtures very out of styrene $\left(F_{S S}=10 \%\right.$ for $\left.f_{a}(0)=83 \%\right)$ (Figure 8$)$.

This low reactivity of the AN involves consequently, obtaining a maximum of alternation for an initial mixture of monomers very strongly charged in $\mathrm{AN}\left(f_{a}(0)=88 \%\right)$. It should be also noted that the value of the maximum of the alternate dyads $\left(\max F_{A S}=85 \%\right)$ is a little higher than for the emulsions with $\mathrm{E} / \mathrm{M}=5$ and $\mathrm{E} / \mathrm{M}=0$; the product $r_{a} . r_{s}$ being smaller for $\mathrm{E} / \mathrm{M}=10$ (Figure 9). 


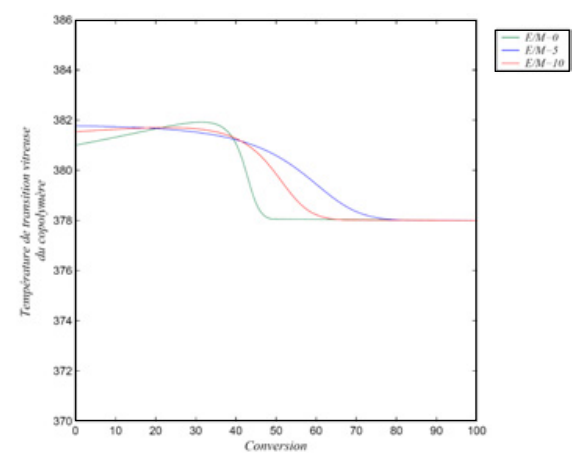

Figure 10. Simulation of copolymerization $A N / S, E / M=$ 0,5 et $10, f_{a}(0)=80 \%$. Evolution of $T_{g}$ according to conversion.

For the initial composition $80 \%$ in $\mathrm{AN}$, the results of kinetics showed a difference in the behavior of the reaction in copolymerization, according to the report $E / M$, where it noted in particular a greater drift of composition according to the dilution of the emulsion thus involving a distribution of the different sequences.

For $E / M=0, E / M=5$ et $E / M=10$, we observe that $T_{g}$ instantaneous macromolecules will correspond to pure polyAcrylonitrile starting from a all the more low conversion as $E / M \uparrow$, involving a formed final total copolymer of a quantity of polyAcrylonitrile pure (Figure 10), the total final copolymer resulting from each one of these reactions of copolymerization will have a field of vitreous transition necessarily different in spite of the same average composition. This illustrates the fact well that if $T_{g}$ initially depends it on the total composition of copolymer, it depends more finely on the distribution of the sequences of the copolymer which is in fact in relation to the process of synthesis (medium homogeneous and report $E / M$ for the emulsion).

\section{CONCLUSION}

These theoretical results are important and will make it possible insofar as they are confirmed by an experimental study, to know a priori the behaviour of the reaction of copolymerization of any system of monomers as well as the microstructure and of $T_{g}$ the macromolecules formants the copolymer, starting from the simulation of this reaction of copolymerization on the basis of relatively simple principle of calculation, knowing the reports of reactivity of the couple of monomers. They constitute a good base for future, theoretical and/or experimental work relating to for example the copolymerization of this system or other, by the process used in industry such as the continuous semi process making it possible to correct the strong drifts of composition observed for certain compositions and to obtain homogeneous copolymers, or to carry out copolymerizations of systems to three monomers (terpolymerisation).

\section{References}

[1] Hatate, T. Hano, T. Miyata, F. Nakasmio, N. Sakai, Kagoku Kagaki, 35, 903 (1971).

[2] Pichot, E. Zaganiaris and A. Guyot, J. Polym. Sci., Symp 52, 55 (1975).

[3] Pham and C. Pichot, Macromol. Chem. 180, 2359 (1979).

[4] Meyer, V. E. and G. E. Lowry, J. Polym. Sci, a3, 2843 (1965).

[5] Dionisio J. M. and K. F. O'driscoll, J. Polym. Sci. Polym. Lett. ed. 17, 701 (1979).

[6] Capek I, V. Juranicova and J. Bartoh, Makromol. Chem. 184, 1597 (1983).

[7] O'driscoll, K. F., L. T. Kale, L. H. Garcia- Rubio and P.M. Reilly, J. Polym. Sci. Polym. Chem. ed. 222777, (1984).

[8] Teramachi, S. A. Hasegawa, F. Sato and N. Takemoto, Macromolécules, 18, 347 (1985).

[9] Stejskal J., P. Kratochvil, D. Strakova and O. Prochazka, Macromolécules, 19, 575 (1986).

[10] Hatate, T. Hano, T. Miyata, F. Nakasmio, N. Sakai, Kagoku Kagaki. 35, 903 (1971). 